A paper to be presented at the 29th Annual Convention of the American Institute of Electrical

Engineers, Boston, Mass., June 25, 1912.

Copyright, 1912. By A. I. E. E.

(Subject to final revision for the Transactions.

\title{
SIMPLIFICATION OF ELECTRO-THERMAL CALCULATIONS; THE WATT AND THERMAL OHM
}

BY CARL HERING

Modern tendencies are toward greater efficiency, in machinery as also in a more scientific management and in many other directions involving the saving of useless labor or wasted efforts, as exemplified for instance in simplified spelling the use of abbreviations, the Esperanto language, etc. Obsolete, old-fashioned and inefficient machinery is willingly discarded for that which is more efficient; obsolete methods of management are discarded for better ones; economy in the physical labor of man is carefully studied and practiced; yet in the very process which every engineer himself uses and which is more personal to him than any other, namely, the process of doing his mental work, the average engineer completely ignores the adoption of more efficient methods even when they are within his reach. In our numerous daily calculations we still adhere with astonishing tenacity to our antiquated, cumbersome, irrational, time robbing, inherited systems of measures, although we have within reach systems which are far more efficient in the economy of mental energy.

We have before us an excellent example in the so-called absolute or c.g.s. system of units, which is so invaluable to the physicist. In this there is always one, and only one, well defined unit for every physical quantity, and practically all the conversion factors fall out of the calculations, as they are unity, because all the units are connected with each other by a unity relation.

We could readily have the benefits of such an ideally simple and time saving system in our daily calculations by merely using as our practical units some fixed decimal multiples of these 
generally inconveniently small or large c.g.s. units. Our wise forefathers gave us some of these benefits when they established our present simple and very satisfactory system of practical electric units in which all relations are unity, and we should be everlastingly thankful to them for their forethought and wisdom.

But realizing the great mental inertia which has barred us in this country from the benefits of a great step in this direction in the form of the metric system, the introduction at the present time of such a more complete and radical change for the better as mentioned above, would seem hopeless. The chief reason is that each individual considers only his own small special field of work; there are too few broad-minded men who can see the matter in the light of the benefit to the community as a whole, and who can forget their own temporary inconvenience.

Although there exists this strong opposition to improving the efficiency of our mental work by changing existing units into more rational ones, there is an opportunity to at least take a step in this direction whenever an occasion arises to create a new unit where none has existed before. In fact in such a case it is, in the writer's opinion, a moral duty to our descendants to make such new units decimal multiples of the c.g.s. units, as those who established our electrical units did.

Such an occasion has arisen lately in connection with the flow of heat through bodies, a branch of applied science to which more attention has been given recently and will undoubtedly be given to a much greater extent in the near future; it has its application in the economy of thermal processes. Heat cannot be confined like electricity by practically perfect insulators; it will flow more or less through all bodies, hence will escape and be lost, thereby decreasing the efficiency of a thermal process. It therefore becomes important to be able to measure, calculate and predetermine such flows, and to do this requires that units be established for quantitatively expressing the physical quantities involved. The very rapid introduction of electric heating is making this important, particularly as its commercial success is dependent so largely, if not almost entirely, on the greater ability to prevent a waste of the energy, the prime cost of which is generally greater than that of combustion heat.

There is some property of heat which causes it to flow, another which offers resistance to such a flow, and as this resistance is different in different materials there is a thermal resistivity of materials; there is therefore also a conductance and conductivity ; 
the quantity of heat must be measurable, as also the current or flow of heat. To express, measure and calculate these quantities intelligently and conveniently, there should be a unit for each.

The purpose of the present article is to point out what the most rational values of these different units are and to urge their general adoption.

The selection of new units should, in the writer's opinion, be governed by two principles; one is to bring the new unit into the group based on the c.g.s. units by making it some decimal multiple of the corresponding unit in that system; and the other is to choose these decimal multiples so that in the most frequently occurring calculations the relations between the different units are unity, just as in our existing system of electrical units, and in the c.g.s. system.

The cause of a flow of heat is a difference of temperature. As there is unfortunately no unit of temperature in the c.g.s. system, we are compelled to adopt one of the thermometer scales and there is presumably no question that among these the centigrade system should be preferred. Hence the unit of difference of temperature which should be used in the present new group of units is the centigrade degree.

For expressing the flow of heat it would at first seem natural to use as a unit a transmission of one of the customary units of heat per second, like calories per second. It would then however not be in that preferred group of rationally interconnected units to which the c.g.s. and the electrical units belong, as there is no heat unit in that system. Heat is only one of the various forms of energy and one of the basic principles of a rational, interconnected system of units, is to have only one unit for each physical quantity no matter what form it is in. The c.g.s. unit of energy is the erg, hence this includes heat as well as all other forms of energy. It is therefore not correct to call a calorie per second a c.g.s. unit, as is not uncommonly done by able writers.

A flow or current of heat is physically a rate of transmission of energy, hence the only rational unit for measuring it in the c.g.s. system is the erg per second. The physicist would use this unit in his analytical deductions. This is more rational also because in many of our processes energy is transformed, sometimes repeatedly, from one form to another, and as it is the same energy it is not rational or mentally economical to keep changing the units in our calculations each time this same energy merely changes its form; the physicist would certainly not do so in an 
analytical discussion, and as his method is far simpler why should not the engineer also avail himself of this privilege; it is directly within his reach.

According to the above principles therefore the practical unit of a flow or transmission of energy, be it in the form of heat or in any other form, should be a decimal multiple of the erg per second. As such a practical unit of a very suitable order of magnitude already exists in practice in the watt, it would be irrational to create a new one. Hence in the simplified system here suggested, the watt is chosen as the rational unit for the flow of heat. It is equal to $10,000,000$ ergs per second.

When it is said that a heat flow of say 10 watts is passing through a thermal conductor, or is flowing out of its end or from a surface, it means that the amount of heat flowing is equal to that which would be generated continuously in an electrical conductor, lamp or coil in which 10 watts of electrical energy is being converted into heat. No conversion factors are then required although the energy has changed from the electrical to the thermal form.

Some persons argue that in this as also in the adoption of the kilowatt in place of the horse power, we electrical engineers want others to adopt our electrical units to the exclusion of the older units, and they ask why we should not change ours to suit the older ones. This is manifestly unjust; what we ask is not that the other units be "electrified," but merely that these scattered, arbitrary, incommensurate and often quite irrational units should be changed so as to bring them into that rational c.g.s. group in which (nearly) all conversion factors disappear, and to which the electrical units already belong; and whenever the magnitude of the practical electrical unit for that quantity is of a convenient order, it certainly simplifies matters to adopt this already existing unit, like the watt in this case, instead of creating another one.

Having selected the centigrade degree as the unit of the cause of thermal flows, or what might popularly be called the thermal pressure, and having chosen the watt as the unit of the flow, then the rational unit of the resistance which opposes such a flow, is that resistance which will allow one watt of heat current to flow through it when the difference in temperature at the ends is one centigrade degree. This means that these three units bear a unity relation to one another, just, as in accordance with Ohm's law, the unit ohm was so chosen that one volt will cause one ampere to flow through it. 
This unit, which was suggested by the writer over a year ago therefore is analogous to the ohm and the calculations involving it are like those for electrical resistances. The thermal resistance of a centimeter or inch cube is the specific resistance or resistivity, and the total resistance is equal to this resistivity multiplied by the ratio of the length to the section.

The flow of heat through a body in watts (assuming it to be perfectly insulated on the lateral surfaces) will be numerically equal to the difference of temperature at its ends, in centigrade degrees, divided by the thermal resistance of the body, provided the unit of resistance just defined is used. That is, if $W$ represents the flow in watts, $T$ the drop of temperature in centigrade degrees and $R$ the thermal resistance in terms of this unit, then $W=T / R$, which on account of its identity with Ohm's law might be termed the thermal Ohm's law, especially as it is believed that Dr. Ohm discovered his famous electrical law through the analogy of the flow of electricity with the flow of heat, the laws of the latter having been determined earlier.

Owing to the analogy between the unit of thermal resistance as thus defined, with the electric ohm, and in deference to Dr. $\mathrm{Ohm}$ who about a century ago first pointed out the analogies between the two laws, the writer suggested calling this unit of thermal resistance the thermal ohm.

When a unit is often used, it facilitates and simplifies matters to give it some distinctive name. It is for instance very convenient to be able to use the simple name " ampere " for the unit of an electric current, instead of its more cumbersome though quite correct equivalent the "volt per ohm." Or to use the name " ohm " in place of "volts per ampere." It seems almost essential to name two of such an interconnected group of three units, then why not name the third one also. Such a distinctive name is also useful to distinguish a unit more readily from other units of the same physical quantity when several are in use, as for instance in the case of feet, meters and inches, or pounds and kilograms, or horsepowers and kilowatts, etc.

Some persons however do not agree with the present writer in the advisability of thus naming this new unit, although they agree that the magnitude of the unit is the best; they prefer to define it each time it is used by stating that a thermal resistance when given in terms of this unit is equal to so-and-so many " degrees per watt," on the same principle that the name of the ohm is unnecessary as this unit could correctly be specified 
as the number of "volts per ampere." Unfortunately neither of these two phrases carries with it the often very useful conception that the physical quantity referred to is a resistance. The writer thinks it better to give it a characteristic name and therefore advocates the term "thermal ohm" as a simple one which carries with it an explanation that it represents a thermal resistance, while the last word " ohm " indicates that it belongs to the same ideal group of units to which the electric ohm belongs, in which the values are always some decimal multiples of the c.g.s. units.

Whenever the difference of temperature between the ends of a thermal conductor, stated in centigrade degrees, is divided by the thermal flow in watts, the quotient, being the degrees per watt, is the thermal resistance in thermal ohms.

The writer acknowledges that Dr. A. E. Kennelly first suggested this appropriate name for a unit of thermal resistance but had applied it to one of a different magnitude, namely the one based on the gram calorie, therefore bringing it into a group which is foreign to and incommensurate with the true c.g.s. group; but as that name for that unit had apparently not come into general use, although it had been suggested a number of years before, the writer took the liberty to retain what he considered a very appropriate name but to change the numerical magnitude of the unit so as to bring it into the more rational group.

That this unit is of a convenient size for use in practical work is shown in various tables of values of the thermal resistivities of various materials* and surfaces $\dagger$ which were gathered from various sources and reduced by the writer to thermal ohms. The metals for instance have thermal resistivities of about onequarter to three thermal ohms for one centimeter cube; solid refractory materials like brick, about 40 to 200 ; loose granular or fibrous materials about 300 to 4000 . Surfaces from which heat is emitted seem to have a surface resistance of several thousand to less than one thermal ohm per square inch, depending upon the nature of the surface and the temperatures.

In thermal problems like in electrical ones, it is often convenient and time saving, in the calculations occurring in practice,

*Flow of Heat through Bodies. Metallurg. \& Chem. Engineering, Dec. 1911, p. 652.

$\dagger$ Flow of Heat Through Contact Surfaces. Metallurg. \& Chem. Engineering, Jan. 1912, p. 40. 
to use the thermal resistances of bodies as resistances and not carry the calculation each time to the extent of determining the loss of heat through it, as was formerly done before thermal resistances came into more general use. This would apply for instance where such resistances are used as thermal insulators. This often saves many unnecessary calculations, and it also eliminates the temperature from such calculations, except in so far as the temperature coefficient of the thermal resistivity is concerned. A wall of a furnace or the thermal insulation of a cooking device can be specified simply as having a resistance of so-and-so-many thermal ohms.

Although in most engineering problems of an electro-thermic nature, like electric heating devices including furnaces, it is far simpler to use resistances instead of conductances, as is also generally true in purely electric problems, yet it may sometimes be desirable to use thermal conductances and conductivities and for this purpose the units for the latter should of course be the true reciprocals of the resistance and resistivity units. For consistency the name "thermal mho" is recommended for the corresponding unit of thermal conductance.

A more detailed discussion of this unit of thermal resistance was published by the writer over a year ago* in which article a complete set of the conversion factors were given for reducing values in other units to those in the units here suggested and the reverse, as also numerical examples illustrating the use of this unit in practice. These conversion factors are as follows:

\section{TABLE OF CONVERSION FACTORS}

(Note.-A flow or radiation of heat may be measured in watts, in gram calories per second, or in ergs per second. In this table the term " c.g.s. unit," that is, the absolute unit, refers to the true ones based on the erg per second; those often called " c.g.s. units " and based on the gram calorie are here given under the latter term. The centigrade degree is understood to be meant in all these units.)

Thermal Resistance.

Thermal ohms:

$=$ centigrade degrees $\div$ watts $=$ degrees per watt.

$=1 \div$ thermal mhos.

1 thermal ohm:

$=$ a resistance requiring $1^{\circ} \mathrm{C}$. per watt of flow.

$=4.18617$ gram calorie units; approx. 50/12.

$=0.0000001$ c.g.s. unit of thermal resistance.

*Thermal Resistance and Conductance; the Thermal Ohm and Thermal Mho. Metallurg. \& Chem. Engineering, Jan. 1911, p. 13. 
1 gram calorie unit:

$=$ a resistance requiring $1^{\circ} \mathrm{C}$. per flow of a gram calorie per second.

$=0.238882$ thermal ohms; approx. 24/100.

$=2.38882 \times 10^{-8}$ c.g.s. units of thermal resistance.

1 c.g.s. unit of thermal resistance:

$=$ a resistance requiring $1^{\circ} \mathrm{C}$. per flow of an erg per second.

$=10,000,000$. thermal ohms.

$=41,861,700$. gram calorie units.

\section{Thermal Conductance.}

Thermal mhos:

$=$ watts $\div$ centigrade degrees $=$ watts per degree.

$=1 \div$ thermal ohms.

1 thermal mho:

$=a$ conductance permitting a flow of 1 watt per degree.

$=0.238882$ gram calorie unit; approx. 24/100.

$=10,000,000$. c.g.s. units of thermal conductance.

1 gram calorie unit:

$=\mathrm{a}$ conductance permitting a flow of 1 gram calorie per second per degree.

$=4.18617$ thermal mhos; approx. 50/12.

$=41,861,700$. c.g.s. units of thermal conductance.

1 c.g.s. unit of thermal conductance:

$=$ a conductance permitting a flow of 1 erg per second per degree.

$=0.0000001$ thermal mho.

$=2.38882 \times 10^{-8}$ gram calorie units.

\section{Thermal Resistivities.}

Note.-These are the reciprocals of the corresponding conductivity units.

1 gram calorie, centimeter cube unit:

$=0.393700$ gram calorie, inch cube, unit; appprox. $4 / 10$.

$=0.238882$ thermal ohm, centimeter cube unit; approx 24/100.

$=0.0940478$ thermal ohm, inch cube, unit; approx. $2 / 21$.

$=2.38882 \times 10^{-8}$ c.g.s. units resistivity.

1 gram calorie, inch cube unit:

$=2.54001$ gram calorie, centimeter cube units; approx. 10/4.

$=0.606762$ thermal ohm, centimeter cube unit; approx. 3/5.

$=0.238882$ thermal ohm, inch cube unit; approx. 24/100.

$=6.06762 \times 10^{-8}$ c.g.s. units of resistivity.

1 thermal ohm, centimeter cube unit:

$=4.18617$ gram calorie, centimeter cube units; approx. 50/12.

$=1.64809$ gram calorie, inch cube units; approx. $5 / 3$.

$=0.393700$ thermal ohm, inch cube units; approx. $4 / 10$.

$=10^{-7}$ c.g.s. unit of resistivity.

1 thermal ohm, inch cube unit:

$=10.6329$ gram calorie, centimeter cube units; approx. 21/2.

$=4.18617$ gram calorie, inch cube units; approx. 50/12.

$=2.54001$ thermal ohm, centimeter cube units approx. 10/4.

$=2.54001 \times 10^{-7}$ c.g.s. units of resistivity. 
1 c.g.s. unit of resistivity:

$=418617 \times 10^{7}$ gram calorie, centimeter cube, units.

$=1.64809 \times 10^{7}$ gram calorie, inch cube units.

$=10^{7}$ thermal ohm, centimeter cube units.

$=3.93700 \times 10^{6}$ thermal ohm, inch cube units.

Thermal Conductivities.

Note.-These are the reciprocals of the corresponding resistivity units.

1 c.g.s. unit of conductivity:

$=2.54001 \times 10^{-7}$ thermal mho, inch cube unit.

$=10^{-7}$ thermal mho, centimeter cube unit.

$=6.06762 \times 10^{-8}$ gram calorie, inch cube unit.

$=2.38882 \times 10^{-8}$ gram calorie, centimeter cube unit.

1 thermal mho, inch cube unit:

$=3.93700 \times 10^{6} \mathrm{c}$.g.s. units of conductivity.

$=0.393700$ thermal mho, centimeter cube unit; approx. 4/10.

$=0.238882$ gram calorie, inch cube unit; approx. 24/100.

$=0.0940478$ gram calorie, centimeter cube unit; approx. $2 / 21$.

1 thermal mho, centimeter cube unit:

$=10^{7}$ c.g.s. units of conductivity.

$=2.54001$ thermal mho, inch cube units; approx. 10/4.

$=0.606762$ gram calorie, inch cube unit; approx. $3 / 5$.

$=0.238882$ gram calorie, centimeter cube unit; approx. $24 / 100$.

1 gram calorie, inch cube unit:

$=1.64809 \times 10^{7}$ c.g.s. units of conductivity.

$=4.18617$ thermal mho, inch cube units; approx. 50/12.

$=1.64809$ thermal mho, centimeter cube units; approx. $5 / 3$.

$=0.393700$ gram calorie, centimeter cube unit; approx. 4/10.

1 gram calorie, centimeter cube unit:

$=4.18617 \times 10^{7}$ c.g.s. units of conductivity.

$=10.6329$ thermal mho, inch cube units; approx. 21/2.

$=4.18617$ thermal mho, centimeter cube units; approx. 50/12.

$=2.54001$ gram calories, inch cube units; approx. 10/4.

As the calculations involving thermal resistances and flows of heat through them become so simple when these units are used, and are identical with the analagous electrical ones, it seems superfluous to give any numerical examples here as illustrations. Such examples are given in some of the papers that have been referred to. Attention however is here called to the fact that although the temperature coefficient of thermal resistivities are probably of about the same general order of magnitude as those of electric conductivities and therefore not large per degree, yet the ranges of temperature are sometimes so great, as in furnaces 
for instance, that these changes should be taken into consideration.*

Besides using watts and thermal ohms to simplify thermal calculations, it will greatly simplify numerous other calculations in electro-thermic work to apply the same reasoning to the heat unit itself, that is, to abandon all the several so-called heat units, namely the two calories, the British thermal unit, and the hybrid pound-centigrade unit, and use the watt-hour instead. This brings the heat unit into the c.g.s. group and relieves all subsequent calculations of the troublesome conversion factors involving the mechanical or electrical equivalents of heat; such conversion factors then become unity.

This is readily done by converting the values of the physical constants of materials given in calories or B.t.u., in the tables, once for all into watt-hours; the numerous electro-thermal engineering calculations based on them then become extremely simple, so simple in fact that many of them can be done mentally.

This would mean converting all such thermal constants as specific heats, latent heats, and heats of chemical combinations, once for all into watt-hours, thereby avoiding the incessant repetition of the reductions involving incommensurate conversion factors in all the numerous subsequent calculations. It would then be stated for instance that the energy necessary to heat, melt and raise the temperature of a pound of steel to a certain number of degrees is so-and-so-many kilowatt hours per pound. This is discussed in another paper by the writer. $\dagger$

The conversion factors are as follows: One kilogram calorie equals 1.1628 or approximately $7 / 6$ watt-hours; one thermal unit (pound-Fahrenheit unit or B.t.u.) equals 0.29303 or approximately $5 / 17$ watt-hours; one watt-hour equals 0.85998 or approximately $6 / 7$ kilogram-calories and to 3.4127 or approximately $17 / 5$ thermal units.

It may at first seem somewhat inconsistent to use the watthour instead of the joule for the equivalent of the heat unit in this simplified system. But although the joule is theoretically the more rational unit, yet electric energy in practice is now universally expressed and measured in watt-hours or kilowatt-

\footnotetext{
*See for instance a recent paper by the author read before the April meeting of the Amer. Electrochem. Society on Effects of the Variations of Thermal Resistivities with the Temperature.

$\dagger$ Paper read before the Amer. Electrochem. Society in April 1912 on "Uniformity and Simplicity in Electrochemical and Electrothermal Calculations."
} 
hours and never in joules, hence by using the former the troublesome factor of 3600 (the number of seconds in an hour) is eliminated by becoming unity.

The simplifications in calculations by thus working within the same group as the c.g.s. units, is so great that it may often reduce the mental labor even in thermal problems which do not involve electrical energy; one would then reduce the original data at the start to this group of units, and the final results back again, which would enable one to make all the calculations involving the design and proportioning, (and these are often by far the more numerous) in the simple system, just as in some classes of work it is simpler to convert the original data into the metric system, then make all the tests and numerous engineering calculations in that system, and convert the final results back into the original units. 